\title{
Radiation induced myxoma of superior vena cava origin presenting as a right atrial mass
}

\author{
Feridoun Sabzi, ${ }^{1}$ Reza Faraji' \\ 'Department of Cardiovascular Surgery, Imam Ali Heart Center, Kermanshah University of Medical Sciences, \\ Kermanshah, Iran.
}

\begin{abstract}
Myxomas are the most common benign cardiac tumors. Myxomas are more common in the left heart chamber than the right side chamber. An extracardiac origin presenting as a right atrial mass is very rare. Right-sided tumors are considerably less common than left-sided tumors, and however myxoma of great vessels origin presenting as right atrial masses are rare but radiation induced villous myxoma in superior vena cava (SVC) is exceedingly rare tumor. A case of radiation induced myxoma originating in a previously undescribed location and presenting as a right atrial mass is reported.
\end{abstract}

Keywords: myxoma; right atrial; vena cava.

\section{INTRODUCTION}

Radio-induced non cardiac myxoma have been known for to occur after treatment of cancer during childhood or adult patients, but cardiac myxoma with extra cardiac origin is exceptional following radiotherapy of metastatic seminoma to skull or mediastinum. ${ }^{1,2}$ The delay of appearance of these tumors, their situation in the fields irradiated and dose received suggests their radio-induced nature. The incidence of radio-induced myxoma in the great vein is extremely rare, and a thorough review of the literature demonstrates no any case in the inferior vena cava (IVC), in the femoral vein, in the superior vena cava (SVC) and other great veins. The following is a report on an interesting case of a radio-induced SVC myxoma following radiotherapy for seminoma.

\section{CASE REPORT}

A 27-year-old man was admitted to our hospital with dyspnea. He had been having progressive dyspnea and episodic syncope over the last month. There were no other symptoms. He has seminoma in stage $2 \mathrm{~A}$ in left testis with involvement of retropritoneum and mediastinum that treated with chemotherapy and a course of $600 \mathrm{gGY}$ radiotherapy four years ago. The patient presented with convulsion three month ago. Radiographic imaging revealed large mass in temporal brains lobe. The patient underwent resection of tumor; pathologic examination confirmed its nature as metastatic seminoma. The patient received another course of radiotherapy $(200 \mathrm{gGY})$ and chemotherapy. Physical examination revealed a slender man with total baldness caused by chemotherapy, a blood pressure of $110 / 80 \mathrm{~mm} \mathrm{Hg}$, a pulse rate of 110 beats $/ \mathrm{min}$ and a grade 2/6 systolic murmur at the left sternal border. Results of liver function tests were within normal limits. Chest radiography showed no abnormalities, and electrocardiography revealed sinus tachycardia. Echocardiography showed that the right atrium was almost completely filled by a mobile, homogeneous mass (Figure 1) that intermittently occluded the tricuspid orifice and appeared to emerging from the area of the

Correspondence: Reza Faraji, Department of Cardiovascular Surgery, Imam Ali Heart Center, Kermanshah University of Medical Sciences, Kermanshah, Iran. E-mail: r.faraji61@gmail. com, Tel: +9809183362603, Fax: +988319360043. 
SVC. The diagnosis of a right atrial myxoma was made and the patient was taken to the operating theater. At median sternotomy, bicaval cannulation could not be safely accomplished because palpation of the superior vena cava (IVC) revealed that the tumor was emerging into the right atrium (RA) from within that vessel. The right innominate vein, inferior vena cava, and aortic root were cannulated. On bypass, the right atrium was opened and a cluster of gripes-shaped, glistening, yellow mass of very firm consistency was noted to be filling it. The mass was not attached to the RA and in fact represented the end of a solid cord of tumor that was emerging from the SVC orifice, and, under tension, could be made to stretch a further $5 \mathrm{~cm}$ into the RA (Figure 2).
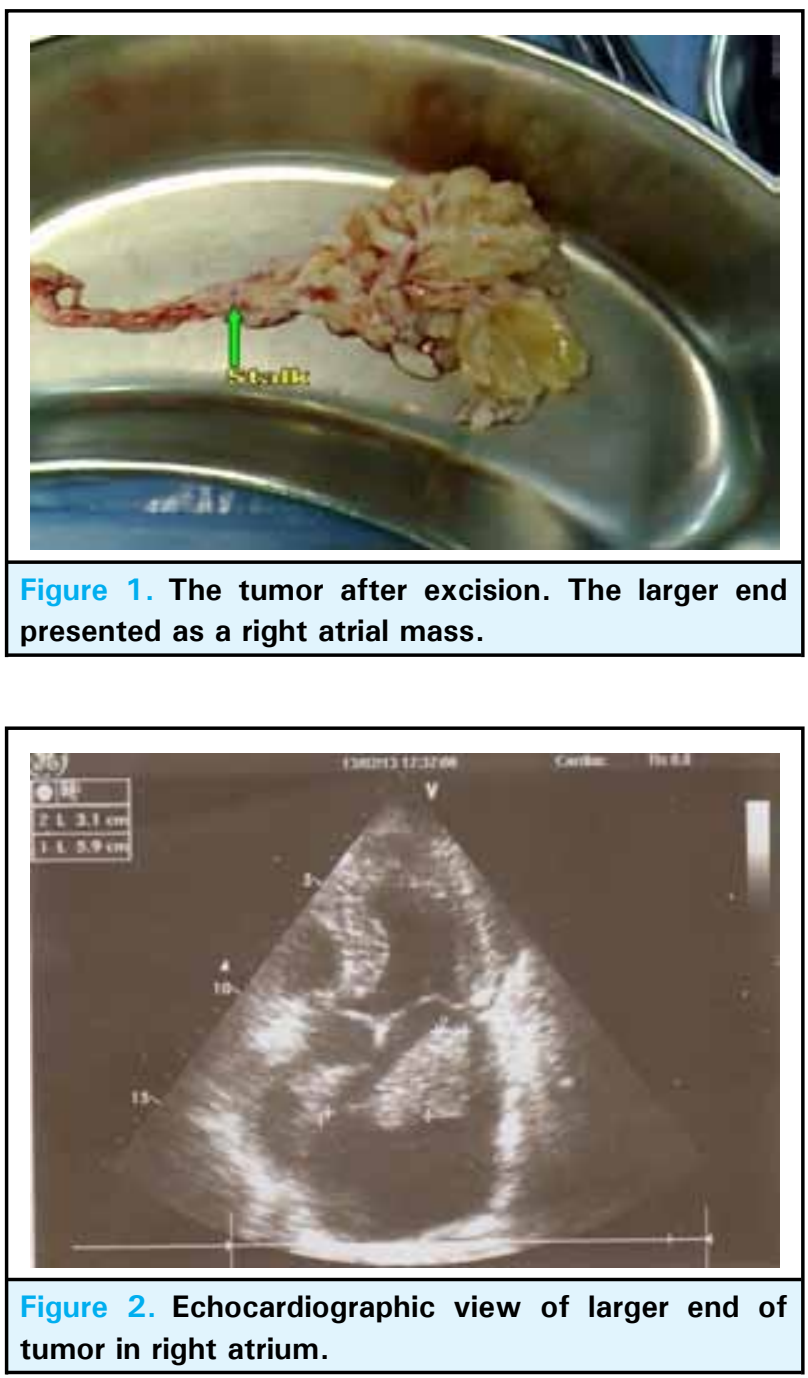

The point of origin could be ascertained in innominateSVC junction and the decision was made to amputate the stalk of the tumor under caudal traction, whereupon the stump, which was approximately $1 \mathrm{~cm}$ in diameter, retracted beyond sight into the innominate vein. The tumor stalk was palpable within the innominate vein and an incision at the site where the tumor was palpable was made and remainder of tumor bases removed. The patient was weaned off bypass uneventfully and her postoperative course was unremarkable. Pathologic examination of the mass revealed a $5 \times 10-\mathrm{cm}$ firm, smooth tumor. Histological examination revealed villous myxoma, with no features of malignancy. Follow-up echocardiography exam showed no recurrences of tumor in innominate vein.

\section{DISCUSSION}

The data on the late cardiovascular toxicity of radiation therapy (RT) come primarily from survivors of breast cancer and Hodgkin lymphoma (HL), diseases in which $\mathrm{RT}$ is a frequent component of the initial management and in which survival is often prolonged. ${ }^{3}$ Daoud et al. found that neoplasm developing within the radiation field (RF) can be radiation induced (RI) or coincidental. ${ }^{4}$ The most common adverse effect of radiation was pericarditis. RI cardiomyopathy has been reported by Hu et al. ${ }^{5}$ but the development of great vessels tumors was exceedingly rare. In a case study, patient developed parotid myxoma three year after radiotherapy of Hodgkin lymphoma. Cahan et al. ${ }^{6}$ established criteria for RI neoplasm as follows: 1- tumor must be appearing in the IF, 2- the tumor is not present prior to irradiation, 3- A sufficiency latency period must be histological proven and must be a different type of tumor from the originally tumor first treated within RF. Our case report meets each of these criteria, because the myxoma occurred within RF four year after radiotherapy, and pathology proven myxoma. Therefore patients undergoing radiation therapy should have a long term follow for probable secondary neoplasm beyond the primary cancer. Radiation induced neoplasm may occur outside of the field of radiotherapy. ${ }^{7}$ The dosage of radiation may play an important role in tumor rose of secondary neoplasm within RF. The present case of secondary myxoma was speculated to be radiation related or spontaneously, SVC endothelial cell mutation. It is not still unknown how long secondary tumors will develop after radiotherapy for seminoma. Radiation induced myxoma presenting as right atrial masses with extra cardiac origin are exceedingly rare, and a literature search reveals no previously reported cases of a similar nature to ours case. Abu-auda et al. ${ }^{8}$ described a case report of myxoma that occurred 21 years after seminoma surgery. The author did not reveal that myxoma induced by radiation. Garick et al. 9 reported a case of cardiac myxoma that developed after radiotherapy of a childhood neuroblastoma. All others myxoma arising from the IVC or from the femoral vein was primary myxoma unrelated to radiation. ${ }^{1,2}$ This patient may be the only known case of a radiation induced myxoma arising from a SVC that presenting as a right atrial mass. Precise localization of the point 
of origin was attempted, because to prevent tumor recurrence, the origin must be completely excised, be it free atrial wall, or innominate wall. Devig et al. ${ }^{10}$ showed that if the tumor cannot be completely excised, a caval umbrella or similar device should be inserted.

\section{CONCLUSIONS}

As this case clearly illustrates, rare and bizarre variations of origin of myxoma may occur and will be discovered before the operation only if an index of suspicion is maintained.

\section{REFERENCES}

1. Cujec B, Ulmer B, Mckaigney JP, Bharadwaj B. Right atrial myxoma presenting as Budd-Chiari syndrome. Ann Thorac Surg. 1987;44:658-9.

2. Bortolotti U, Faggian G, Mazzucco A. Right atrial myxoma originating from the inferior vena cava. Ann Thorac Surg. 1990;49:100-2.

3. Mueller OM, Van De N. Surgical treatment of primary intracranial myxoma in a child following radiotherapy: case report and review of the literature. Childs Nerv Syst. 2010;26:829-34.

4. Daoud J, Ben Salah H, Kammoun W, Ghorbel A, Frikha M, Jlidi $\mathrm{R}$, et al. Radiation-induced glioblastoma and myxoma after treatment for undifferentiated carcinoma of the naspharynx. Cancer Radiother. 2000;4:469-72.
5. Hu JQ, Guan YH, Zhao LZ. Delayed radiation encephalopathy after radiotherapy for nasopharyngeal cancer. J Comput Assist Tomogr. 1991;15:181-7.

6. Cahan WG, Woodard HQ, Higinbotham NL. Sarcoma arising in irradiated bones. Cancer. 1948;1:29-3.

7. Schrantz JL, Araoz CA. Radiation induced meningeal fibrosarcoma. Arch Pathol. 1972;93:31-26.

8. Abo-Auda WS, Chidambaram BS, Baker K, Whitaker I. Ventricular myxoma presenting as acute visual loss. Tenn Med. 1998;91:391-2.

9. Garick H, Sharon C, Derek W. Cardiac myxoma after treatment for childhood. Neuro Ped Cardio. 2009;30:340.

10. Devig GPM, Clark TA, Aaron BL. Cardiac myxoma arising from the inferior vena cava. Chest. 1980;78:784-6. 